\title{
Petrous Bone Cholesteatoma: Classification, Management and Review of the Literature
}

\author{
Mete Iseri ${ }^{1}$, Sebla Calıskan ${ }^{2}$ and Ahmet Kara ${ }^{3 *}$ \\ ${ }^{1}$ Department of Otorhinolaryngology, Medical Park Hospital, Kocaeli, Turkey \\ ${ }^{2}$ Department of Otorhinolaryngology, Derince Training and Research Hospital, Kocaeli, Turkey \\ ${ }^{3}$ Department of Otorhinolaryngology, Sakarya University Faculty of Medicine, Sakarya, Turkey
}

Submission: June 26, 2018; Published: July 18, 2018

*Corresponding author: Ahmet Kara, Sakarya University Training and Research Hospital, 54000, Korucuk, Sakarya, Turkey, Tel: +90 530 7716317;

Email: doktor.kbb@hotmail.com

\begin{abstract}
Introduction: Petrous bone cholesteatoma Petrous bone cholesteatoma $(\mathrm{PBC})$ is defined as cholesteatoma extending medial to otic capsule. Because of its relations with vital structures, surgical management is generally difficult. Objective: In this study, we aim to share our clinical experiences about surgical approaches, managements and follow up results in petrous bone cholesteatoma.

Methods: A retrospective clinical study was conducted on patients who had been diagnosed as PBC between 2003 and 2012 years. Sanna classification was used for the pathology extending. A total of 13 patients with 14 ears were evaluated about symptoms, clinical and radiological findings, surgical techniques, complications and follow up results.

Results: The mean age of patients was 33,3 years ranging from 18 to 52 years. Hearing loss was the most common symptom (92\%). Five patients had facial paralysis preoperatively. Massive type was the most common type. Different surgical techniques were carried out for each pathological type. Mean follow up time was 34,3 months. Recurrence was identified in one patient twice within two years and revision surgical procedures were performed.

Conclusion: Extent of the PBC should be well defined in preoperative imaging and type of surgical approach should be planned before with an appropriate classification. Regular follow up is mandatory for patients with MRI every year.
\end{abstract}

Keywords: Petrous bone; Cholesteatoma; Hearing loss

\section{Introduction}

Petrous bone cholesteatoma (PBC) is defined as cholesteatoma located medial to the otic capsule. It is an uncommon pathology and because of functional damages in the treatment it diminishes the patients' quality of life [1]. Diagnosis and surgical approach may be challenging. Advanced imaging techniques are necessary for assessment. This lesion generally has a slow-growing behavior, so it may remain asymptomatic for years. It can be congenital or acquired. Our aim with this study is to report our management and surgical experiences of fourteen cases diagnosed with PBC.

\section{Material and Methods}

This is a retrospective clinical trial. Inclusion criteria were the patients had been operated with petrous bone cholesteatoma and had long-term follow-up results. Patients with limited cholesteatoma, and recently operated were excluded. Patients diagnosed with and operated from PBC between 2003 and 2012 were analyzed retrospectively. The Ethics Committee of our institution approved the study (2015/87) and written informed consent was taken. A total of 14 cases (13 patients) were included in this study. All patients underwent through clinical otoneurological and audiologic examinations. All patients evaluated by preoperative computed tomography (CT), temporal bone magnetic resonance imaging (MRI) and/or if necessary magnetic resonance venography (MRV) imaging. House Brackmann (H-B) classification system was used to evaluate the facial nerve functions. Our surgical approaches, outcomes, recurrences, complications and follow up results were evaluated with the review of the literature.

\section{Results}

Patients consisted of 12 men and one woman. One patient had bilateral PBC. Mean age was 33.3 years ranging from 18 to 52. All patients were adults and patients diagnosed as acquired cholesteatoma had chronic ear disease like otorrhea, tympanic membrane retraction or perforation. Only one patient had intact tympanic membrane and no history of ear disease. Five of them had surgery for cholesteatoma before. Postoperative 


\section{Global Journal of Otolaryngology}

follow-up period was on average 34.3 months ranging from 12 months to 8 years. The most common symptom was hearing loss $(92 \%)$ followed by otorrhea (78\%). Other symptoms were facial paralysis (35\%), dizziness (35\%), tinnitus and headache in descending order of frequency. Ear side numbers were equal (7 left sided, 7 right sided) (Table 1). Two patients had cerebellum abscess and sigmoid sinus thrombosis preoperatively. Sanna classification [2] was used to evaluate the petrous bone cholesteatomas (Table 2). Massive type was the most common type in our study and supralabyrinthine, infralabyrinthine and apical type (Figures 1-4) were observed respectively (Table 3). Surgical approaches performed for the patients are listed in Tables 4 \& 5. Recurrence was seen in one patient twice within two years and revision surgical procedures were performed. Facial paralysis was observed in five patients preoperatively. According to $\mathrm{H}-\mathrm{B}$ classification system, grade II facial palsy was detected in one patient, grade III in one patient and grade VI facial palsy in three patients. Facial nerve could not be protected in patient who had one and a half months history of grade III facial palsy, so facial nerve grafting was performed for the patient. Facial nerve was decompressed in patient with grade II. The remaining three patients who had fibrotic nerve and long duration of palsy received static reconstruction of the face.

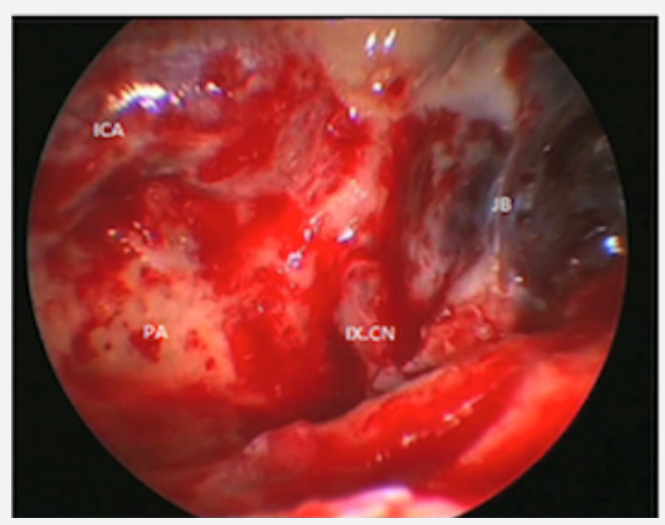

Figure 1: Apical type PBC. Cholesteatoma located petrous apex is marked with white arrow, also seen clivus extension at medial.

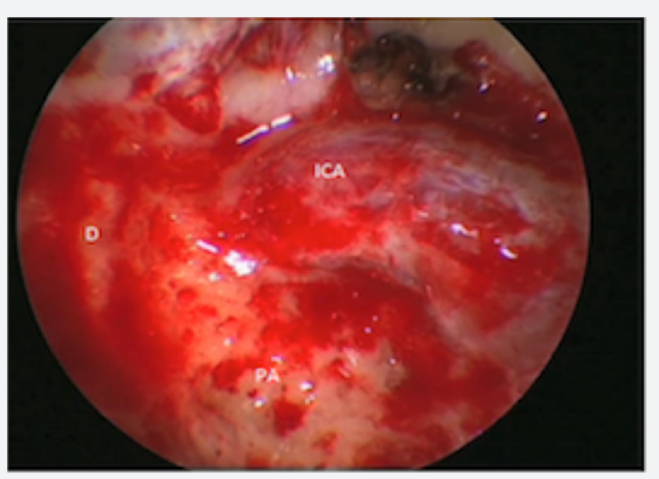

Figure 2: CT image of the patient that had bilateral petrous bone cholesteatoma. Especially on the right, it is seen that the massive type of cholesteatoma eroded the dura.

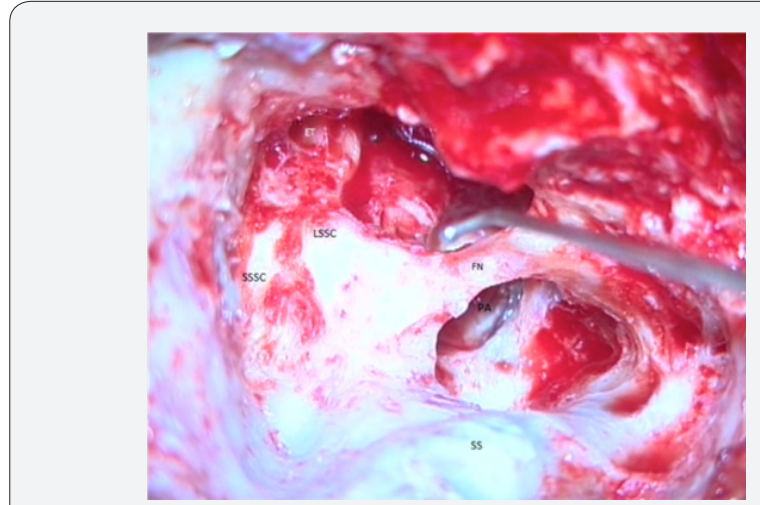

Figure 3: MR image of the patient that had bilateral petrous bone cholesteatoma. Indicated by the white arrow on the left cholesteatoma located superior to the labyrinth. Supralabyrinthine type petrous bone cholesteatoma.

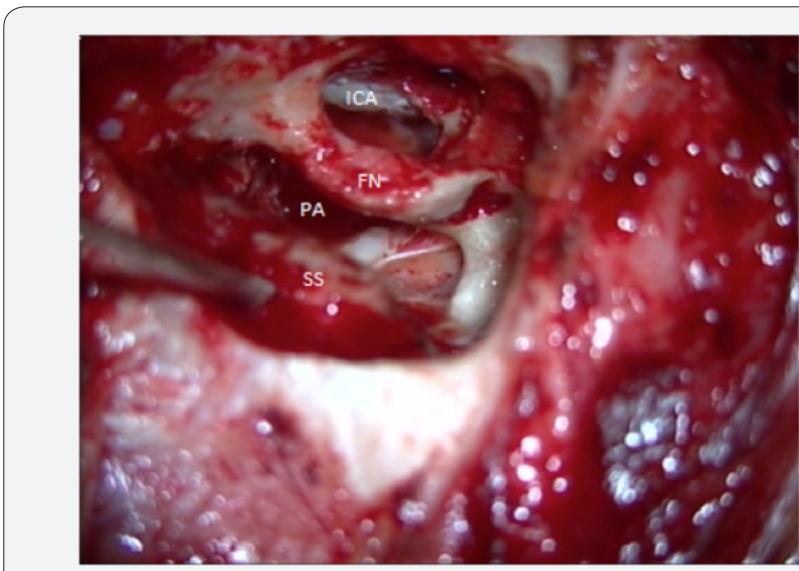

Figure 4: Coronal section of $\mathrm{CT}$, infralabyrinthine type.

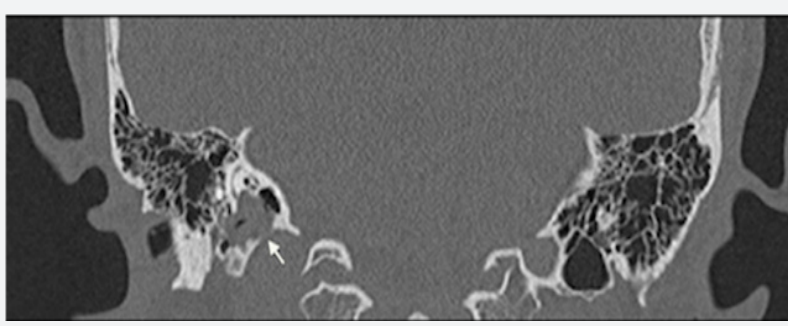

Figure 5: Massive type cholesteatoma exsicion by transotic approach. FN: Facial nerve, PA: Petrous apex, SS: Sigmoid sinus, ICA: Internal Carotid Artery.

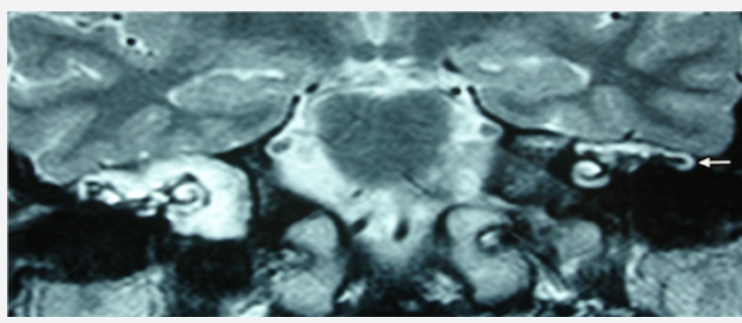

Figure 6: Subtotal Petrosectomy. LSSC: Lateral Semisircular canal, SSSC: Superior Semisircular Canal, ET: Eustachian Tube, FN: Fasiyal Nerve, ICA: İnternal Carotid Artery, PA: Petrous Apex, SS: Sigmoid Sinus. 


\section{Global Journal of Otolaryngology}

In terms of the surgical approach to remove the cholesteatoma matrix; modified trans cochlear approach type A (MTCA) was used for three patients, enlarged trans labyrinthine approach (ETLA) for four patients, transotic approach (TO) for three patients (Figure 5), modified trans cochlear approach type B (MTCB) for one patient, combined middle fossa transmastoid approach for one, transmastoid retrolabyrinthine approach for one patient and subtotal petrosectomy (Figure 6) operation for

Table 1: Clinical findings of 13 patients with petrous bone cholesteatoma.

\begin{tabular}{|c|c|c|c|c|c|c|}
\hline Case no & Age (years) & Sex & Side & Symptoms & Etiology & H-B Grade \\
\hline 1 & 34 & M & $\mathrm{L}$ & HL1, otorrhea & Acquired & \\
\hline 2 & 30 & M & $\mathrm{L}$ & HL, otorrhea & Congenital & \\
\hline 3 & 26 & M & $\mathrm{L}$ & HL, FP2, otorrhea & Recurrent/Iatrogenic & Grade III \\
\hline 4 & 50 & M & $\mathrm{L}$ & HL, FP, otorrhea, dizziness & Acquired & Grade VI \\
\hline 5 & 18 & M & $\mathrm{L}$ & HL, otorrhea & Recurrent/Iatrogenic & \\
\hline 6 & 23 & M & $\mathrm{R}, \mathrm{L}$ & HL, FP, otorrhea & Recurrent/Iatrogenic & Grade VI \\
\hline 7 & 52 & M & $\mathrm{R}$ & HL, FP, otorrhea, dizziness & Acquired & Grade II \\
\hline 8 & 50 & M & $\mathrm{R}$ & HL, otorrhea & Acquired & \\
\hline 9 & 41 & M & $\mathrm{R}$ & $\mathrm{HL}, \mathrm{FP}$ & Acquired & Grade VI \\
\hline 10 & 23 & $\mathrm{~F}$ & $\mathrm{R}$ & HL,otorrhea,dizziness, tinnitus & Acquired & \\
\hline 11 & 36 & M & $\mathrm{R}$ & HL, otorrhea & Acquired & \\
\hline 12 & 25 & M & $\mathrm{L}$ & HL, dizziness & Acquired & \\
\hline 13 & 26 & M & $\mathrm{R}$ & Dizziness & Acquired & \\
\hline
\end{tabular}

1. Hearing Loss

2.Facial Paralysis

Table: 2

\begin{tabular}{|c|c|}
\hline Class & Spread \\
\hline \multirow{4}{*}{ Class I: Supralabyrinthine } & Anterior: horizontal part of Intrenal carotid artery (ICA) \\
\hline & Posterior: posterior bony labyrinth \\
\hline & Medial: Internal acoustic canal (IAC), petrous apex \\
\hline & Inferior: basal turn of the cochlea \\
\hline \multirow{4}{*}{ Class II: Infralabyrinthine } & Anterior: ICA vertical part, petrous apex, clivus \\
\hline & Posterior: dura of the posterior cranial fossa and sigmoid sinus \\
\hline & Medial: IAC, lower clivus, occipital condyle. \\
\hline & Inferior: jugular bulb, lower cranial nerves \\
\hline \multirow{4}{*}{ Class III: Infralabyrinthine - Apical } & Anterior: ICA vertical \pm horizontal parts \\
\hline & Posterior: posterior fossa through the retrofacial air cells \\
\hline & Medial: petrous apex, clivus, sphenoid sinus, rhinopharynx \\
\hline & Inferior: jugular bulb, lower cranial nerves \\
\hline \multirow{4}{*}{ Class IV: Masivve } & Anterior: ICA vertical \pm horizontal parts \\
\hline & Posterior: posterior fossa dura and IAC \\
\hline & Medial: petrous apex, superior and midclivus, sphenoid sinus \\
\hline & Inferior: infralabyrinthine compartment \\
\hline \multirow{4}{*}{ Class V: Apical } & Anterior: Meckel's cave area and may involve the V. Nerve \\
\hline & Posterior: IAC and posterior cranial fossa \\
\hline & Medial: superior or mid clivus, sphenoid sinus \\
\hline & Inferior: infralbyrinthine compartment \\
\hline
\end{tabular}

one patient were performed. We performed the right subtotal petrosectomy in a patient with infralabyrinthine type. This surgical procedure included after canal wall down mastoidectomy and complete drilling of the air cells of the temporal bone was carried out, the cholesteatoma sac was followed and retrofacial, retrolabyrinthine, infralabyrinthine, peritubal, and pericarotid air cells were removed and only a few cells in the petrous apex left behind. 


\section{Global Journal of Otolaryngology}

Table 3: Sanna Classification of Petrous Bone Cholesteatoma.

\begin{tabular}{|c|c|}
\hline Subclass & Features \\
\hline Clivus (C) & $\begin{array}{c}\text { superior and mid clival extensions are seen } \\
\text { from massive, infralabyrinthine - apical } \\
\text { and apical PBC whereas the lower clival } \\
\text { involvement is a feature of infralabyrinthine- } \\
\text { apical PBC }\end{array}$ \\
\hline Sphenoid sinus (S) & $\begin{array}{c}\text { sphenoid sinus involvement is seen from } \\
\text { anteromedial extensions of massive, } \\
\text { infralabyrinthine apical and apical PBC; it is a } \\
\text { rare extension }\end{array}$ \\
\hline Rhino pharynx (R) & $\begin{array}{c}\text { it is the rarest extension of the PBC; it is an } \\
\text { extension of infralabyrinthine- apical or } \\
\text { massive PBC, which may extend through the } \\
\text { clivus beneath the sphenoid sinus into the } \\
\text { rhino pharynx. }\end{array}$ \\
\hline
\end{tabular}

Table 4: Classification of PBC versus pathologic type.

\begin{tabular}{|c|c|c|c|c|}
\hline Pathologic type & Congenital & Acquired & $\begin{array}{c}\text { Recurrent/ } \\
\text { Iatrogenic }\end{array}$ & Total \\
\hline Supralabyrinthine & 0 & 4 & 0 & 4 \\
\hline Infralabyrinthine & 0 & 3 & 0 & 3 \\
\hline $\begin{array}{c}\text { Infralabyrinthine- } \\
\text { apical }\end{array}$ & 0 & 0 & 0 & 0 \\
\hline Massive & 0 & 3 & 3 & 6 \\
\hline Apical & 1 & 0 & 0 & 1 \\
\hline
\end{tabular}

Table 5: Surgical approach and class of PBC.

\begin{tabular}{|c|c|c|}
\hline Surgical Approach & No. Of Patients & Classification \\
\hline TO & 3 & 2 Massive - 1 Apical \\
\hline ETLA & 4 & 2 SL-2 IL \\
\hline SP & 1 & IL \\
\hline MF+TM & 1 & SL \\
\hline TM+RL & 1 & SL \\
\hline MTCA & 3 & Massive \\
\hline MTCB & 1 & Massive \\
\hline
\end{tabular}

TO: transotic; ETLA: enlarged trans labyrinthine approach; SP subtotal petrosectomy; MF+ TM: Middle Fossa + trans mastoid approach; TM+RL: Transmastoid+Retrolabyrinthine; MTCA: modified trans cochlear approach type A; MTCB: Modified trans cochlear approach type B; SL: Supralabyrinthine; IL: Infralabyrinthine.

\section{Discussion}

The largest series of petrous bone cholesteatoma reported in the world literature to date belongs to Sanna et al. with 129 cases [2]. Incidence of PBC in their series was $2.9 \%$. Omran et al had 93 cases of PBC in their study [3]. In our retrospective study with 14 cases we evaluated our management, results and postoperative follow-up experiences accompanied by literature knowledge. According to the Sanna classification massive type was the most common type and hearing loss was the most common symptom in our study similar to the series of Omran et al. PBC treatment is surgery and cholesteatoma needs to be removed clearly. Its extension should be evaluated before the surgery so imaging techniques have critical roles on management of PBC. Computed tomography and MRI are the best modalities for definition of petrous apex lesions, differential diagnosis, or making a decision for surgical approach and to see the recurrences. In MRI cholesteatoma can be distinguished from the cholesterol granuloma by the presence of intermediate-to-low T1-weighted signal, from a mucocele by the presence of restricted diffusion on diffusion weighted images, and from a benign bone tumor by the absence of enhancement on contrast enhanced images [4]. In some extensive cases sigmoid sinus obliteration could be necessary by intraluminal or extraluminal, so the dominant sinus identification must be done before the surgery. Therefore, MR venography can make a contribution to treatment plan. As Pandya et al [5] described before, some points have to be taken into considerations when treating patients. These are

\section{i. Complete eradication of the disease}

ii. Preservation of facial nerve function

iii. Prevention of CSF leakage and meningitidis; and

iv. Cavity obliteration. Hearing preservation is not the primary aim of treatment after radical removal of cholesteatoma. Surgical approaches for PBC can vary from subtotal petrosectomy to modified trans cochlear approaches $[2,3,5]$ including lateral transtemporal (trans labyrinthine, transcochlear, infralabyrinthine, infracochlear) and middle fossa approaches. Furthermore, use of intraoperative navigational systems can lead combination of an endoscopic transsphenoidal approach with transtemporal or middle fossa (MF) approaches [6].

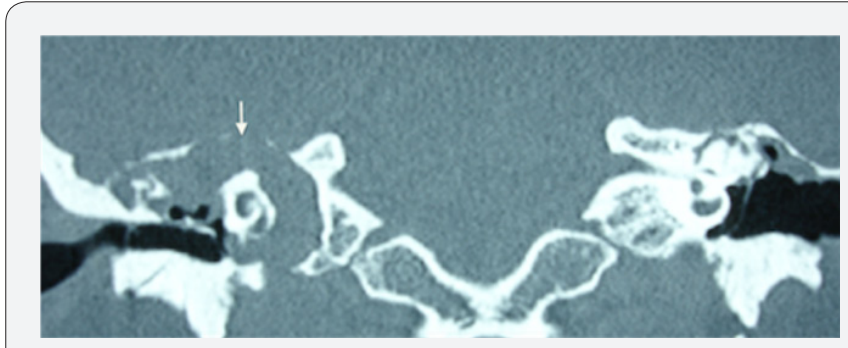

Figure 7: Modified Transcochlear Approach type A. Endoscopic examination of right ear. D: Dura, ICA: İnternal carotid artery, PA: Petrous Apex.

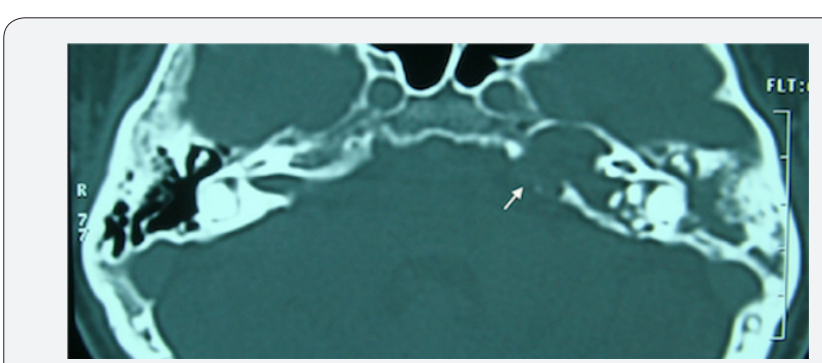

Figure 8: Right ear Modified Transcochlear Approach surgery endoscopic view. ICA: İnternal carotid artery, JB: Jugular Bulb, IX. CN: IX. cranial nerve, PA: Petrous Apex.

In massive type $\mathrm{PBC}$, we preferred transotic approach or modified trans cochlear approaches type A and type B (Figures 7-8). Facial nerve function was normal preoperatively in transotic approach group. Sanna et al described in their study an algorithm 


\section{Global Journal of Otolaryngology}

for management of the PBC [2]. Our approach was similar to them about massive type cholesteatoma surgery depending on facial nerve function. Although kinds of surgical techniques have come up with this entity, recurrence is still a big problem. The most important reasons for this problem are incomplete removal of cholesteatoma because of critical structures like dura, facial nerve, carotid artery etc. and inadequate consideration of secret areas. Therefore, an endoscopic examination with 30 degree and/or 45 degree endoscopes should be done after removing the cholesteatoma in terms of residual cholesteatoma. Generally, it is hard to preserve hearing level in transotic approach, trans labyrinthine or trans cochlear approaches. The aim of complete eradication of the disease necessitates sacrifying the labyrinth. We could preserve hearing in only three patients. One of them underwent middle (MF) fossa and trans mastoid approach (TM); one of trans mastoid and retro labyrinthine and the other one underwent subtotal petrosectomy. Patient with bilateral PBC had massive type in his right ear and supralabyrinthine type in left ear. Combined MF-TM approach was used for the only hearing ear and MTCA for the other one. Senn et al. [7] reported in their study that hearing preservation was feasible [7]. Kim et al. [8] reported in their study that preserving the hearing level was possible in supralabyrinthine type with appropriate surgery. It is possible to have control on cholesteatoma extending from medial side of superior semicircular canal to internal acoustic canal and geniculate ganglion by middle fossa and trans mastoid approach together, so we had this operation in one patient mentioned above. Infralabyrinthine type cholesteatoma usually extends from medial side of semicircular canals to internal acoustic canal along the posterior cranial fossa dura or extends to petrous apex from medial side of facial nerve and inferior cochlea between the jugular bulb and internal carotid artery. It is possible to remove cholesteatoma and preserve the hearing by subtotal petrosectomy or trans mastoid retro labyrinthine approach for these selected patients.

Patients may suffer for prolonged facial paralysis in extensive cases. Axon et al noted that significant preoperative paresis or paralysis was almost always associated with erosion at the geniculate ganglion, which suggested ischemic etiology. Danesi et al. [9] reported in their series labyrinthine segment was the most frequent site of injury conversely Sanna and Tutar et al. [10] Among the five patients with facial paralysis, only three had prolonged facial asymmetry history. The remaining two patients had grade II and grade III facial paralysis that had been occurred recently. One was treated by extended trans labyrinthine approach with decompressing the nerve. In the patient with grade III facial paralysis, cholesteatoma was extending to medial to labyrinth and internal acoustic canal invading the facial nerve in labyrinthine and intracanalicular segment. Facial nerve could not be preserved, and great auricular nerve graft was used for rehabilitation. One year after graft surgery patient had minimal movement in his left mouth angle. Facial nerve was considered as fibrotic in three patients with massive type PBC so static reconstruction was carried out for these patients. End-to-end hypoglossofacial anastomosis is still effective procedure in facial reanimation surgery [11]. Generally open radical cavity is favorable and conventional procedure for cholesteatoma surgery however, cavity obliteration technique is recommended in PBC surgery [12]. A deep cavity forms by subtotal petrosectomy, TO, Infratemporal fossa approach and modified trans cochlear approaches so it creates a risk for facial nerve and vascular structures. Obliteration of cavity with abdominal fat also provides to recognize the recurrences and makes the revisions easier.

\section{Conclusion}

It is hard to manage the cholesteatoma located in petrous part of temporal bone because of vital structures. So that, advanced imaging techniques are required for describing the pathology and planning the surgical approach. In addition, a proper classification depending on extent of pathology should be done before the surgery and patient should be informed about surgical outcomes. Finally, appropriate surgical technique should be chosen, and patients have to be followed up every year with MRI because of asymptomatic recurrences.

\section{References}

1. Magliulo G (2007) Petrous bone cholesteatoma: clinical longitudinal study. Eur Arch Otorhinolaryngol 264(2): 115-120.

2. Sanna M, Pandya Y, Mancini F, Sequino G, Piccirillo E (2011) Petrous bone cholesteatoma: classification, management and review of the literature. Audiol Neurotol 16(2): 124-136.

3. Omran A, De Donato G, Piccirillo E, Leone O, Sanna M (2006) Petrous bone Cholesteatoma: managements and outcomes. Laryngoscope 116(4): 619-626.

4. Connor SE, Leung R, Natas S (2008) Imaging of the petrous apex: a pictorial review. Br J Radiol 81: 427-435.

5. Pandya Y, Piccirillo E, Mancini F, Sanna M (2010) Management of complex cases of petrous bone cholesteatoma. Ann Otol Rhinol Laryngol 119(8): 5d14-525.

6. Aubry K, Kovac L, Sauvaget E, Tran Ba Huy P, Herman P (2010) Our experience in the management of petrous bone cholesteatoma. Skull Base 20(3): 163-167.

7. Senn P, Haeusler R, Panosetti E, Caversaccio M (2011) Petrous bone cholesteatoma removal with hearing preservation. Otol Neurotol 32(2): 236-241.

8. Kim MJ, An YS, Jang MS, Cho YS, Chung JW (2014) Hearing and facial function after surgical removal of cholesteatomas involving petrous bone. Clin Exp Otorhinolaryngol 7(4): 264-268.

9. Axon PR, Fergie N, Saeed SR, Temple RH, Ramsden RT (1999) Petrosal cholesteatoma: management Considerations for minimizing morbidity. Am J Otol 20(4): 505-510.

10. Danesi G, Cooper T, Panciera DT, Manni V, Cote DW (2016) Sanna classification and prognosis of cholesteatoma of the petrous part of the temporal bone: A retrospective series of 81 patients. Otol Neurotol 37(6): 787-792.

11. Catli T, Bayazit YA, Gokdogan O, Goksu N (2010) Facial reanimation with end- to-end hypoglossofacial anastomosis: 20 years' experience. J Laryngol Otol 124(1): 23-25.

12. Moffat D, Jones S, Smith W (2008) Petrous temporal bone cholesteatoma: a new classification and long-term surgical outcomes. Skull Base 18(2): 107-115. 
CC Commons Attribution 4.0 License

BY

DOI: 10.19080/GJO.2018.16.555945
Your next submission with Juniper Publishers will reach you the below assets

- Quality Editorial service

- Swift Peer Review

- Reprints availability

- E-prints Service

- Manuscript Podcast for convenient understanding

- Global attainment for your research

- Manuscript accessibility in different formats

( Pdf, E-pub, Full Text, Audio)

- Unceasing customer service

Track the below URL for one-step submission https://juniperpublishers.com/online-submission.php 